\title{
Interlaboratory Comparison of Backscatter Coefficient Estimates for Tissue-Mimicking Phantoms
}

\author{
Janelle J. Anderson, ${ }^{1}$ Maria-Teresa Herd, ${ }^{1}$ Michael R. King, ${ }^{2}$ Alexander Haak, ${ }^{2}$ \\ Zachary T. Hafez, ${ }^{2}$ Jun Song, ${ }^{2}$ Michael L. Oelze, ${ }^{2}$ Ernest L. Madsen, ${ }^{\prime}$ \\ James A. Zagzebski, William D. O’Brien, JR., and Timothy J. Hall \\ ${ }^{1}$ University of Wisconsin-Madison \\ Wisconsin Institutes for Medical Research \\ 1111 Highland Ave. \\ Madison, WI 53705 \\ tjhall@wisc.edu \\ ${ }^{2}$ Bioacoustics Research Laboratory \\ Department of Electrical and Computer Engineering \\ University of Illinois at Urbana-Champaign \\ 405 N. Mathews \\ Urbana, IL 61801
}

\begin{abstract}
Utrasonic backscatter is useful for characterizing tissues and several groups have reported methods for estimating backscattering properties. Previous interlaboratory comparisons have been made to test the ability to accurately estimate the backscatter coefficient (BSC) by different laboratories around the world. Results of these comparisons showed variability in BSC estimates but were acquired only for a relatively narrow frequency range, and, most importantly, lacked reference to any independent predictions from scattering theory. The goal of this study was to compare Faran-scattering-theory predictions with cooperatively-measured backscatter coefficients for low-attenuating and tissue-like attenuating phantoms containing glass sphere scatterers of different sizes for which BSCs can independently be predicted. Ultrasonic backscatter measurements were made for frequencies from 1 to $12 \mathrm{MHz}$. Backscatter coefficients were estimated using two different planar-reflector techniques at two laboratories for two groups of phantoms. Excellent agreement was observed between BSC estimates from both laboratories. In addition, good agreement with the predictions of Faran's theory was obtained, with average fractional (bias) errors ranging from 8-14\%. This interlaboratory comparison demonstrates the ability to accurately estimate parameters derived from the BSC, including an effective scatterer size and the acoustic concentration, both of which may prove useful for diagnostic applications of ultrasound tissue characterization.
\end{abstract}

KEY WORDS: Backscatter; interlaboratory comparison; tissue characterization.

\section{INTRODUCTION}

The ultrasonic backscatter coefficient (BSC) is a useful property for characterizing tissues. It is defined as the differential scattering cross section per unit volume for a scattering angle of $180^{\circ} .{ }^{1}$ Like most ultrasonic tissue-characterization parameters, the BSC is most useful when it can be estimated with high accuracy and precision. A commonly-used reference for comparison of BSC estimate accuracy and precision is Faran's scattering theory. ${ }^{2}$ Faran's work predicts scattering of plane waves from a single, rigid spherical or cylindrical scatterer, assuming the scatterer is within an inviscid medium. Many studies have tested Faran's theory against experimentally-determined BSC estimates using test phantoms. For example, Burke et $\mathrm{al}^{3}$ showed that despite Faran's inviscid medium assumption, scattering measurements for a phantom with a single steel sphere within a viscous, agar gel background could 
be done and resulted in angle-dependent scattering estimates in good agreement with Faran's prediction. Similar experiments with phantoms that contained narrow distributions of glass bead scatterers produced scattering measurements with comparable accuracy and precision. ${ }^{4}$

Furthermore, ultrasonic backscatter measurement results from phantoms containing either relatively narrow or relatively broad size distributions of spherical scatterers embedded within various materials (agar or gelatin) are consistent with Faran's predictions. ${ }^{1,5-8}$ Additional phantom studies revealed that the BSC could be accurately determined independently of transducer-to-scattering-volume distance, opening up the possibility of an instrument-independent method for determining BSCs. ${ }^{5,6,9}$ Further phantom-based studies demonstrated the ability to obtain BSC estimates and even to generate BSC images up to $12 \mathrm{MHz}$ using a variety of array transducers on clinical scanners. ${ }^{12,13}$ In order to determine the diagnostic capabilities of the BSC and further improve BSC estimation, estimation accuracy must be demonstrated among research groups active in this area, despite differences in methodologies. To do this, several interlaboratory studies were completed to compare consistency of ultrasonic backscatter, attenuation and sound speed measurements of phantoms. The first comparison showed that attenuation estimates among groups agreed, but variable results existed for sound speed and backscatter estimates. ${ }^{12}$ A later comparison demonstrated that BSC estimation was still highly variable in absolute magnitude but the frequency dependencies agreed. ${ }^{13}$

These interlaboratory comparisons were limited in several ways. First, groups participating in these studies obtained measurements over a relatively narrow frequency range of 1 to $9 \mathrm{MHz}$. Second, no comparison with scattering theory was conducted to examine BSC-estimate accuracy. Third, BSC-estimate comparisons were made for phantoms with relatively low $k a$ (product of the wave number and scatterer radius). The largest reported was $k a \cong 1.6$.

The goal of this study was to compare BSC accuracy using well-characterized phantoms of tissue-like attenuating and low-attenuating materials with moderately low to high $k a$ (about 0.01 to 4 ). The phantoms contained spherical glass-bead scatterers with known size distributions allowing accurate predictions of the BSC using Faran's theory. The ultrasonic backscatter from all of the phantoms was measured over a slightly-broader frequency range of 1 to $12 \mathrm{MHz}$ at two different laboratories: the Ultrasound Research Group at the University of Wisconsin-Madison (UW) and the Bioacoustics Research Laboratory at the University of Illinois at Urbana-Champaign (UIUC).

\section{METHODS}

\section{Materials}

Two different groups of phantoms were created: phantoms with low attenuation (LA) and phantoms with tissue-like attenuation (TLA). One LA phantom contained $41 \mu \mathrm{m}$ glass spheres in agar. These spheres had a very narrow distribution of scatterer diameters ( $41 \pm 2$ $\mu \mathrm{m})$ and low $k a$ at the frequencies employed. A second LA phantom $(150-180 \mu \mathrm{m})$ had a broader distribution of scatterer diameters $(160 \pm 60 \mu \mathrm{m})$ and higher $k a$ (Table 1). Seven TLA phantoms were used, all of which had relatively low $k a$, but the diameter distribution and number density of scatterers within each phantom differed (Table 2) as did the attenuation coefficient. Note in table 2 that the composition of the F1 and F2 TLA phantoms differed only in number density ( 2 and $8 \mathrm{~g} \mathrm{cc}^{-1}$, respectively); the M1 and M2 TLA phantoms differed from the M3 TLA phantom only in attenuation $\left(0.45,0.45\right.$ and $0.7 \mathrm{~dB} \mathrm{~cm}^{-1} \mathrm{MHz}^{-1}$, respectively); and the A1 and A2 TLA phantoms were very similar to the F phantoms, except that a narrower distribution of scatterer diameters was used $(45-53 \mu \mathrm{m})$. 
TABLE 1 Low attenuation (LA) phantoms and their compositions. Both phantoms were made with the same weakly-scattering agar background material but contained different sizes and types of scatterers.

\begin{tabular}{ccc}
\hline & $\mathbf{1 5 0 - 1 8 0} \boldsymbol{\mu \mathbf { m }}$ glass beads in agar & $\mathbf{4 1} \boldsymbol{\mu \mathbf { m } \text { glass beads in agar }}$ \\
\hline Number density $(\mathbf{g} / \mathbf{L})$ & 2.23 & 2.23 \\
Sphere type & $3000 \mathrm{E}$ & Duke $41 \mu \mathrm{m}$ \\
Sphere diameter $(\mu \mathrm{m})$ & $150-180$ & $41 \pm 2$ \\
Background material & $2 \%$ agar & $2 \%$ agar \\
Phantom density $(\mathbf{g} / \mathbf{c c})$ & 1.00 & 1.00 \\
Sound speed $(\mathbf{m} / \mathbf{s})$ & 1535 & 1539 \\
Attenuation $(\mathbf{d B} / \mathbf{c m} / \mathbf{M H z})$ & $\sim 0.6$ & 0.1 \\
\hline
\end{tabular}

TABLE 2 Tissue-like attenuation (TLA) phantoms and their compositions. Note that the difference between the F phantoms is their sphere number densities; the difference between the M1/M2 phantoms and the M3 phantom is their attenuation values; and the difference between the A phantoms is their sphere number densities. The differences between the $\mathrm{F}$ and $\mathrm{A}$ phantoms are the sphere size distributions.

\begin{tabular}{|c|c|c|c|c|c|c|}
\hline & F1 & $\mathbf{F} 2$ & M1/M2 & M3 & A1 & A2 \\
\hline Number density (g/L) & 2 & 8 & 4 & 4 & 2 & 8 \\
\hline Sphere type & $3000 \mathrm{E}$ & $3000 \mathrm{E}$ & $3000 \mathrm{E}$ & $3000 \mathrm{E}$ & $3000 \mathrm{E}$ & $3000 \mathrm{E}$ \\
\hline Sphere diameter $(\mu \mathrm{m})$ & $5-40$ & $5-40$ & $5-40$ & $5-40$ & $45-53$ & $45-53$ \\
\hline Background material & $\begin{array}{l}3: 1 \text { gel to } \\
\text { milk }\end{array}$ & $\begin{array}{l}3: 1 \text { gel to } \\
\text { milk }\end{array}$ & $\begin{array}{l}3: 1 \text { gel to } \\
\text { milk }\end{array}$ & $\begin{array}{l}3: 1 \text { gel to } \\
\text { milk }\end{array}$ & $\begin{array}{l}3: 1 \text { gel to } \\
\text { milk }\end{array}$ & $\begin{array}{l}3: 1 \text { gel to } \\
\text { milk }\end{array}$ \\
\hline Phantom density (g/cc) & 1.04 & 1.04 & 1.04 & 1.04 & 1.04 & 1.04 \\
\hline Sound speed $(\mathrm{m} / \mathrm{s})$ & 1552 & 1553 & $1549 / 1552$ & 1559 & 1548 & 1549 \\
\hline Attenuation (dB/cm/MHz) & 0.5 & 0.5 & 0.45 & 0.70 & 0.45 & 0.48 \\
\hline
\end{tabular}

The phantoms were cylindrically shaped (Fig. 1) and were constructed as described by Madsen et al. ${ }^{14}$ The background material for the TLA phantoms was a mixture of gelatin and ultrafiltered milk, whereas the LA phantoms had a weakly scattering $2 \%$ agar background. Glass-sphere scatterers were used in all of the phantoms and are described in table 3 (Potters Industries, Inc., Valley Forge, PA; Thermo Fisher Scientific (formerly Duke Scientific), Inc., Waltham, MA). The distributions of scatterer diameters for each sphere type were measured manually using optical microscopy ${ }^{4}$ and are presented as histograms in figures 2,3 and 4 .

\section{UW experimental procedures}

\section{Sound speed and attenuation}

Sound speed and attenuation coefficients were measured using a narrow band throughtransmission technique ${ }^{14}$ at $22^{\circ} \mathrm{C}$ (Fig. 5). Measurements were performed at five discrete frequencies between 2.25 and $10 \mathrm{MHz}$ using the paired transducers described in table 4 .

A Wavetek (now Willtek Communications, Parsippany, NJ) model 81 function generator was used to excite the transducer with narrow band (30-40 cycle) tone bursts. A transducer 


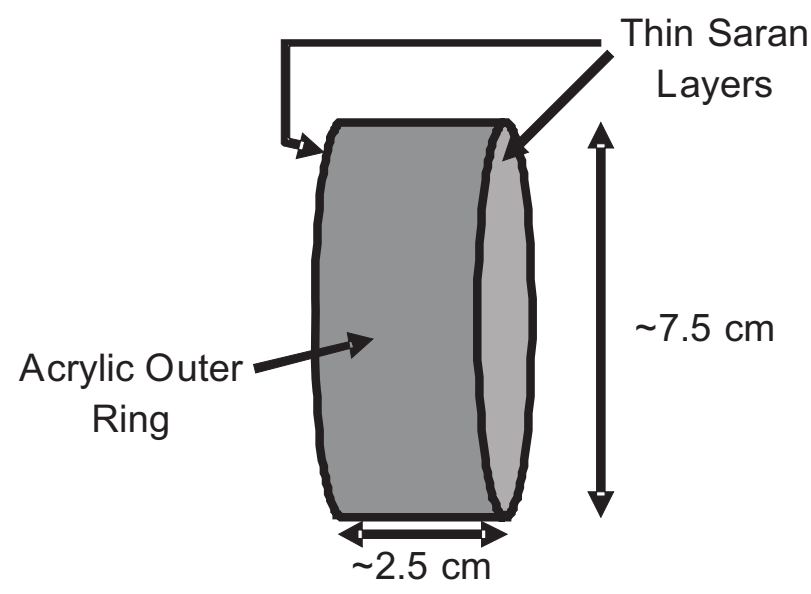

FIG. 1 Schematic of phantom shape, dimensions and construction materials.

TABLE 3 Characteristics of the spherical scatterers used in the phantoms.

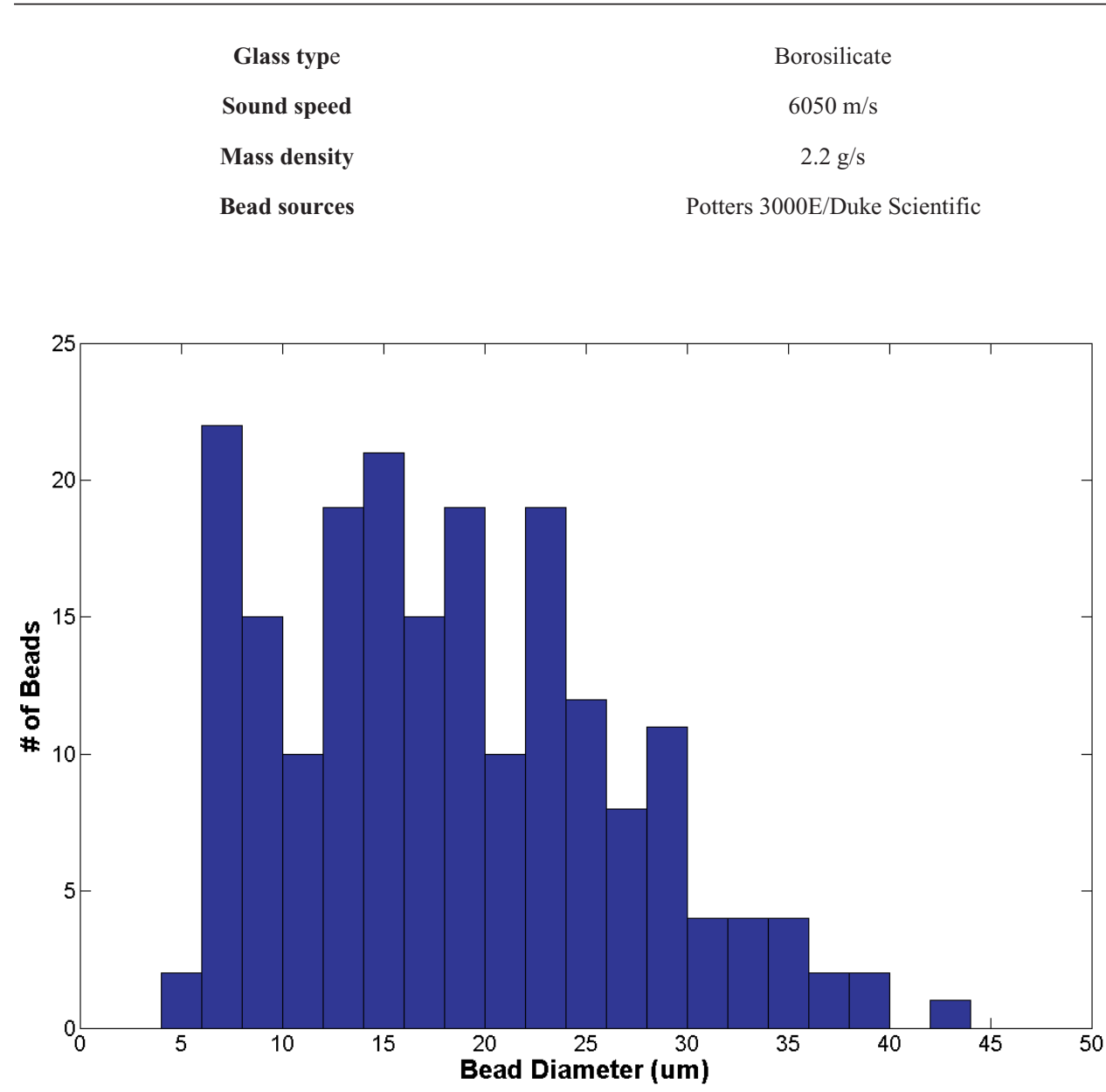

FIG. 2 Diameter distribution for Potters 3000E spheres. These spheres were used in the F1, F2, M1, M2 and M3 TLA phantoms. 


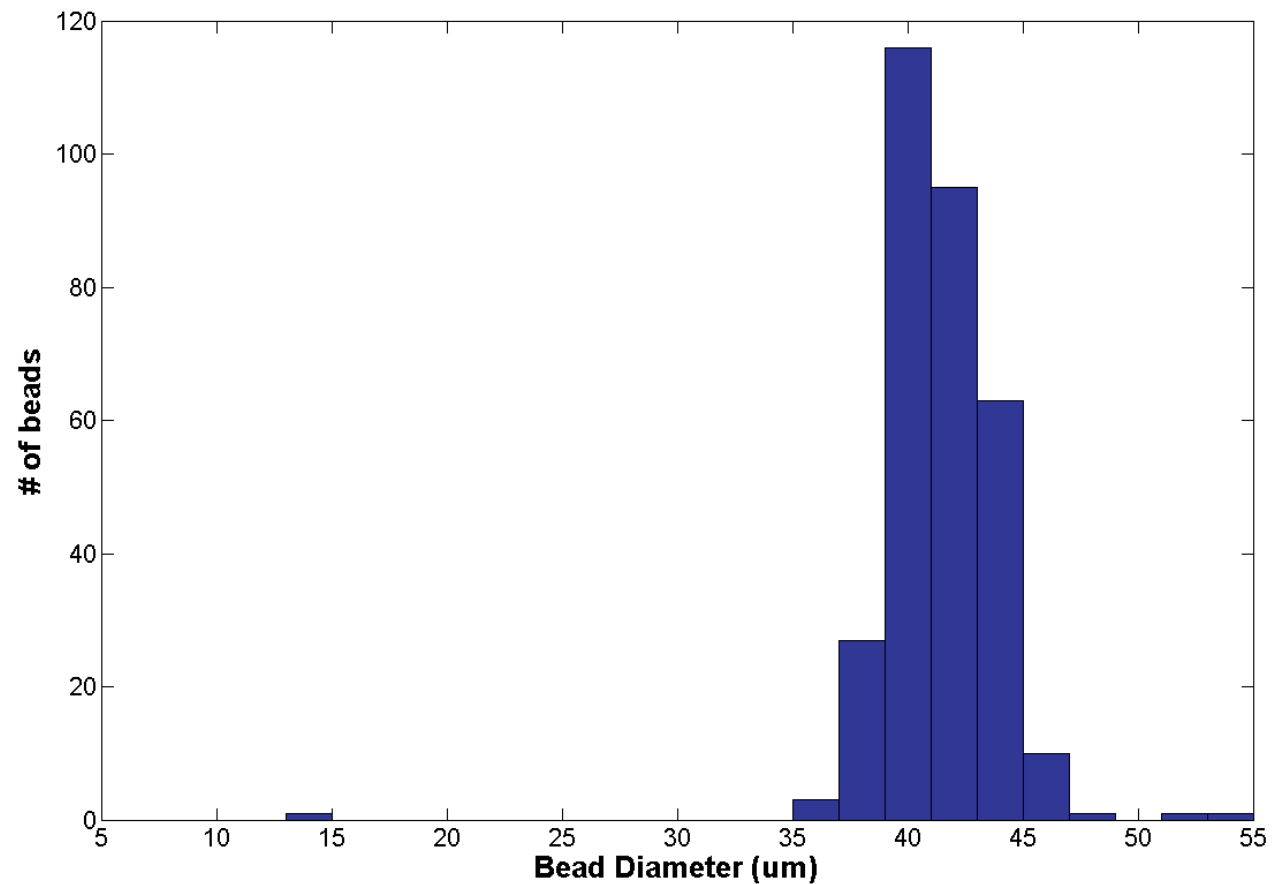

FIG. 3 Diameter distribution for Duke Scientific $41 \mu \mathrm{m}$ spheres. These spheres were used in the $41 \mu \mathrm{m}$ glass spheres in the agar LA phantom.

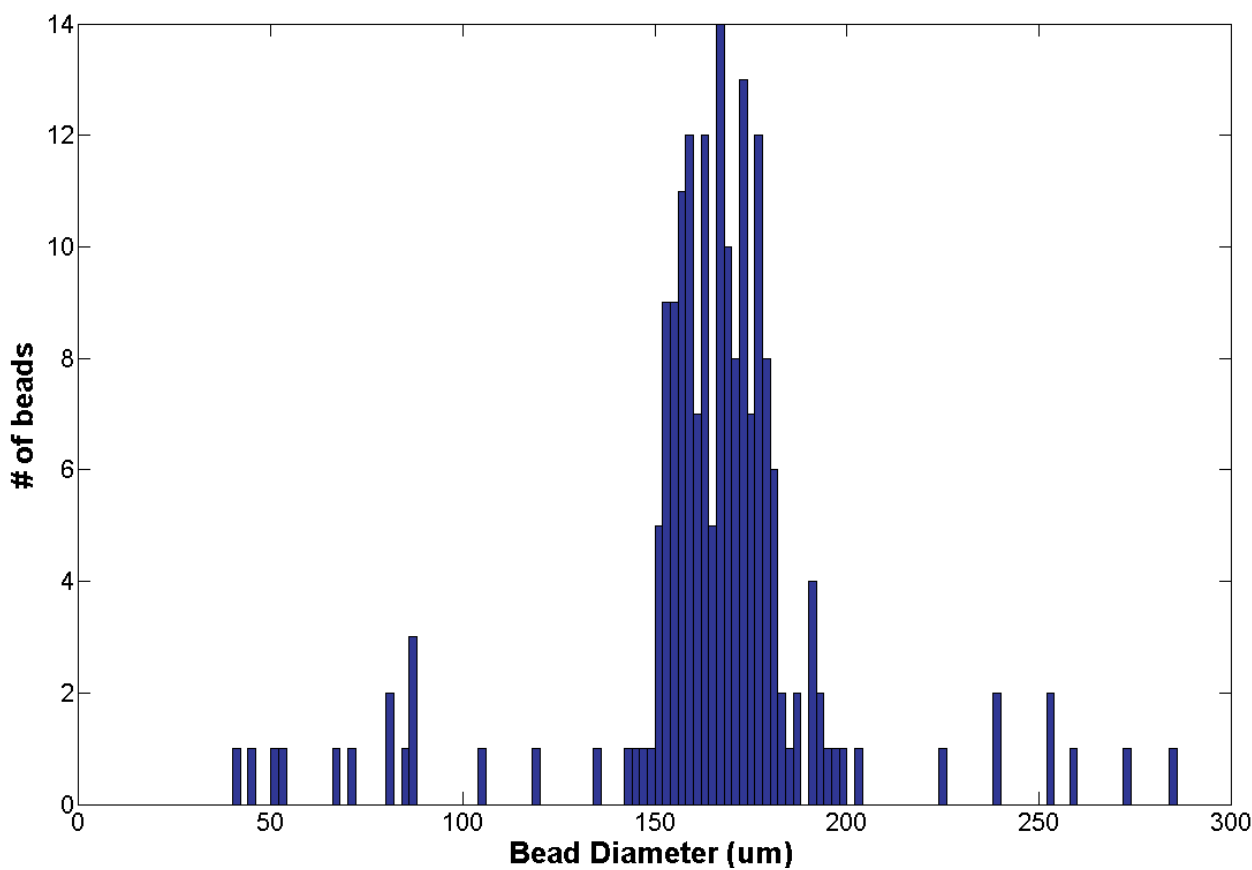

FIG. 4 Diameter distribution for Potters 3000E spheres sieved to 150 to $180 \mu \mathrm{m}$. These spheres were used in the $150-180 \mu \mathrm{m}$ glass spheres in the agar LA phantom. 


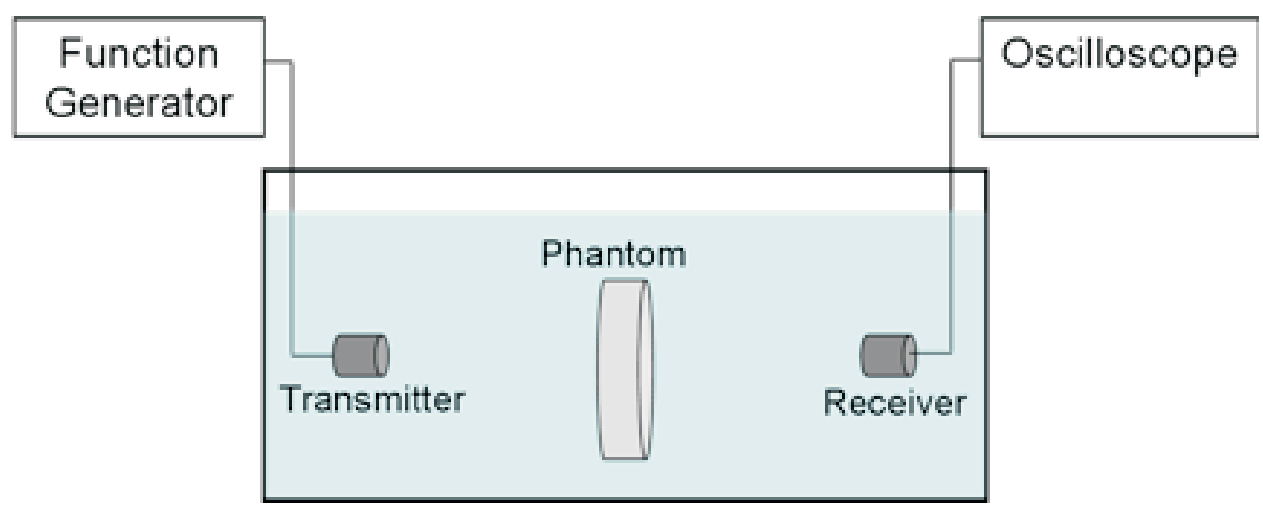

FIG. 5 Setup for through-transmission sound speed and attenuation measurements.

TABLE 4 Transducer information for UW and UIUC sound speed and attenuation measurements.

\begin{tabular}{ccccc}
\hline Institution & $\begin{array}{c}\text { Nominal center } \\
\text { frequency (MHz) }\end{array}$ & $\begin{array}{c}\text { Aperture } \\
\text { diameter }(\mathbf{m m})\end{array}$ & $\begin{array}{c}\text { Focal length } \\
(\mathbf{m m})\end{array}$ & Make, Model \\
\hline UW & 2.25, transmitter & 12.7 & & Panametrics, V306 \\
UW & 2.25, receiver & 6.35 & KB Aerotech, PN2794-1 \\
UW & 3.5, transmitter & 12.7 & Panametrics, V382 \\
UW & 3.5, transmitter & 6.35 & KB Aerotech, PN279-1 \\
UW & 3.5, receiver & 12.7 & Panametrics, V309 \\
UW & 5, transmitter & 6.35 & KB Aerotech, PN2794-3 \\
UW & 5. receiver & 12.7 & Panametrics, V320 \\
UW & 7.5, transmitter & & KB Aerotech, PN2794-4 \\
UW & 7, receiver & 12.7 & Panametrics, V311 \\
UW & 10, transmitter & 6.35 & KB Aerotech, 389039680 \\
UIUC & 10, receiver & 25.4 & 19.1 & Panametrics, V320 \\
UIUC & 8.4, transmitter & $1 \mathrm{~mm}{ }^{2}$ PVDF membrane & Marconi, Y-34-6534 \\
\hline UIUC & hydrophone receiver & & 95 & \\
\hline
\end{tabular}

that matched the frequency response of the transmitting transducer was used to detect the transmitted waveform. The signal from the detector was monitored using a LeCroy model LT342 oscilloscope (LeCroy Corporation, Chestnut Ridge, NY), and time-shifts and amplitude changes with and without the phantom in the transmitter-to-receiver path were manually recorded. Sample thicknesses were measured using a vernier caliper.

Speeds of sound in the phantoms were determined from the measured time-shifts using the same techniques as in reference 12 . Attenuation coefficients were determined by correcting the amplitude changes for effects of reflective losses at the sample-to-water interfaces, correcting for the transmission loss through the thin Saran layers and then expressing the attenuation coefficient at each frequency in $\mathrm{dB} / \mathrm{cm}$. Quadratic curve fitting over the frequencies used was done to determine the attenuation coefficient vs. frequency slope. 
TABLE 5 Transducer information for UW and UIUC BSC measurements.

\begin{tabular}{|c|c|c|c|c|}
\hline Institution & $\begin{array}{c}\text { Nominal center } \\
\text { frequency }(\mathrm{MHz})\end{array}$ & $\begin{array}{c}\text { Analysis } \\
\text { bandwidth (MHz) }\end{array}$ & f-number & Focal length (mm) \\
\hline UW & 3.5 & $2.4-4.9$ & 4.8 & 95 \\
\hline UW & 5 & $3.0-8.0$ & 2.9 & 54 \\
\hline UW & 7.5 & $4.5-9.0$ & 5.0 & 94 \\
\hline UW & 10 & $4.9-13$ & 2.7 & 51 \\
\hline UIUC & 1 & $0.9-1.3$ & 3 & 57 \\
\hline UIUC & 2.25 & $1.5-3.2$ & 2.67 & 51 \\
\hline UIUC & 3.5 & $1.2-5.0$ & 3 & 57 \\
\hline UIUC & 5 & $3.5-6.4$ & 3 & 57 \\
\hline UIUC & 7.5 & $4.1-10$ & 4 & 76 \\
\hline UIUC & 10 & $6.5-14$ & 4 & 51 \\
\hline UIUC & 13 & $9-18$ & 3 & 38 \\
\hline
\end{tabular}

\section{Backscatter coefficient}

To measure the backscatter coefficient, a water tank, LeCroy model LT342 oscilloscope (LeCroy Corporation, Chestnut Ridge, NY), Panametrics 5800 pulser/receiver (Olympus, Waltham, MA), Unidex 11 stepper motor control unit (Aerotech Inc., Pittsburgh, PA), and four spherically-focused transducers (Table 5) were used. The pulser/receiver excites the transducer by using broadband negative spike excitation. The apparatus provided digitized rf echo signals sequenced from different locations in the sample.

To acquire data, the surface of each phantom was aligned at the transducer focus with the Saran surface perpendicular to the beam. This was verified using vertical and horizontal scans relative to the ultrasound beam, determining whether the distance to the surface remained constant. Once the phantom was aligned, a gate duration of $10 \mu \mathrm{s}$ was applied after a delay of $4 \mu \mathrm{s}$. The $4 \mu \mathrm{s}$ delay avoided specular reflections from the phantom surface. Also, low-amplitude excitation and short path lengths in water were used to minimize nonlinear propagation. The phantom was then raster scanned in one plane using a $40 \mathrm{~mm}$ by $40 \mathrm{~mm}$ area at $4 \mathrm{~mm}$ steps, for a total of 121 data steps. At each step, 100 samples of the digitized rf echo signal were averaged to reduce electronic noise. The signal was then stored for later analysis. Echo signals were also acquired from a quartz planar reflector with almost exactly the same setup and equipment settings used for acquiring echo data from the phantoms, except that $40 \mathrm{~dB}$ of attenuation was introduced in the receiver to avoid saturating the echo signal of the planar reflector. The planar reflector face was placed at the focus of the transducer and oriented perpendicular to the beam by maximizing the received signal amplitude across the planar reflector. The received echo signal was averaged over 100 waveforms and stored for later analysis.

Data were analyzed employing the method described by Madsen et al. ${ }^{1}$ A Hamming window was applied to the rf echo signal data from the sample and the power spectrum was estimated from the 121 waveforms. This was divided by a reference spectrum derived from the quartz planar reflector signal. Further details of the analysis, including correcting for transmitted and receiving transducer properties and for beam profiles to account for equipment properties, were the same as described by Madsen et al. ${ }^{1}$ 


\section{UIUC experimental procedures}

\section{Sound speed and attenuation}

All measurements were performed in degassed water at $22 \pm 0.2{ }^{\circ} \mathrm{C}$ using a setup similar to that of UW. The propagation speed and attenuation coefficient values were estimated in through-transmission using the arrival time difference (via a correlation method ${ }^{15}$ ) and the insertion loss techniques, respectively. ${ }^{13}$ All measurements were compensated for the transmission coefficient through the surfaces of the samples.

Spherically-focused transducers were used for sound speed and attenuation estimates and functioned as the source transducers for the propagation speed and attenuation coefficient estimates and for the pulse-echo backscatter coefficient estimates. Source transducers were driven by either a Panametrics 5800 pulser/receiver (Olympus, Waltham, MA) or a Panametrics 5900 pulser/receiver (Olympus, Waltham, MA). The transducers' characteristics were measured by a standard technique and are described in table $4 .{ }^{17}$ The focus of the transmit transducer was positioned with a computer-controlled, motion-control system (Parker-Hannifin Corporation, Harrisburg, PA) on the membrane hydrophone to yield maximum amplitude response.

To measure sound speed, arrival times of received broadband pulses were measured with and without the sample in the water path. Group velocity, $c_{g}$, was computed from

$$
c_{g}=\frac{c_{w}}{1+\frac{c_{w} \Delta t}{d}}
$$

where $d$ is the thickness of the sample, $\Delta t$ is the difference in arrival times and $c_{w}$ is the speed of sound in distilled water. The transit time difference $(\Delta t)$ was measured via a standard correlation-integral technique using Matlab's xcorr function between the water-path and through-sample signals collected at the hydrophone. ${ }^{15}$

The attenuation coefficient was determined from the insertion-loss power spectrum divided by the thickness of the sample. The insertion loss power spectrum $(\mathrm{dB})$ was determined from the difference of two power spectra, the power spectrum of the received $\mathrm{rf}$ waveforms that propagated through water alone and the power spectrum of the received $\mathrm{rf}$ waveforms that propagated through the interposed phantom sample. The insertion loss was divided by the sample thickness $(d)$ and by the frequency to yield the attenuation coefficient (in $\mathrm{dB} \mathrm{cm}^{-1} \mathrm{MHz}^{-1}$ ) as a function of frequency.

\section{Backscatter coefficient}

For BSC estimation, the phantoms were ultrasonically scanned with between 3 and 7 spherically-focused, single-element transducers (Table 5). The scans were conducted in a tank filled with degassed water. A Panametrics model 5900 pulser/receiver was used to excite the transducers. A 200-MHz 14-bit A/D converter (Signatec PDA14-200 A/D converter) combined with a customized LabView oscilloscope program were used to record the waveforms. A motion-control system (Parker-Hannifin Corporation, Harrisburg, PA) was used for scanning.

The scanning procedure for each transducer and phantom began by performing a planar reference scan. Echo signals from a water-Plexiglas interface were recorded over the axial range for which the reflection was greater than half the reflected magnitude at the focus; within this range, the reflected signal was recorded at half-wavelength intervals. Next, the transducer focus was positioned below the surface of the phantom at a distance of half the 
TABLE 6 Sound speed estimates in $\mathrm{m} / \mathrm{s}$. The uncertainty in narrowband sound speed estimates by UW is $\pm 1 \mathrm{~m} / \mathrm{s}$. 19

\begin{tabular}{|c|c|c|c|c|c|c|c|c|c|}
\hline & F1 & F2 & M! & M2 & M3 & A1 & A2 & $\begin{array}{c}41 \mu \mathrm{m} \text { glass } \\
\text { spheres in agar }\end{array}$ & $\begin{array}{l}150-180 \mu \mathrm{m} \text { glass } \\
\text { spheres in agar }\end{array}$ \\
\hline UW & 1552 & 1553 & 1550 & 1553 & 1560 & 1549 & 1550 & 1541 & 1540 \\
\hline UIUC & 1529 & 1530 & 1530 & 1532 & 1561 & 1530 & 1535 & 1566 & 1550 \\
\hline
\end{tabular}

length of the ROI to be used for processing plus $1 \mathrm{~mm}$. A raster scan was then performed, with scan lines recorded at intervals equal to half of the beam width. Generally, the scan covered a sufficient length so that several ROIs could be extracted and processed from each scan.

The BSCs were computed from the raw scan data using the method of Insana and Hall. ${ }^{16}$ This method accounts for equipment-dependent effects by dividing the power spectral magnitude of the echo signals from the phantom by those of the reference (Plexiglas) signals. A correction was made for the layer of Saran covering the phantom. ${ }^{12}$ Also, attenuation was compensated for by assuming that the attenuation had a linear dependence on frequency.

\section{Error analysis}

Average fractional (bias) errors in BSC estimates were calculated over the measured frequency range for each phantom by comparing the UW and UIUC measurements to values predicted with Faran's theory. The error estimates were performed by fitting each group's measurements to a polynomial, subtracting the difference between the Faran predicted and the fitted BSC values, dividing this difference by the Faran-predicted BSC to obtain the absolute fractional difference and averaging all of these absolute fractional differences to obtain the average fractional (bias) error for each group and for each phantom.

\section{RESULTS}

\section{Sound speed}

Sound speed results are shown in table 6 . Sound speed measurements differed from 0.5 to $12 \mathrm{~m} \mathrm{~s}^{-1}$ from the mean of the measurements for each phantom, for a root-mean-square difference of $9.4 \mathrm{~m} \mathrm{~s}^{-1}$.

\section{Attenuation}

Attenuation measurements by the two groups agreed closely, with mean-square differences of $0.01 \mathrm{~dB} \mathrm{~cm}^{-1} \mathrm{MHz}^{-1}$. Attenuation measurements for the LA $41 \mu \mathrm{m}$ glass spheres in agar phantom and for the TLA M2 Phantom are shown in figure 6.

\section{Backscatter coefficient}

Backscatter coefficients measured in both labs are shown in figures 7-13. In each plot, values of the BSC vs. frequency are presented along with the Faran-predicted backscatter displayed as a smooth solid black curve. The other curves represent estimates of BSC from measurements by UW (red lines) and UIUC (blue lines). Different line styles correspond to specific transducers used for data acquisition. 

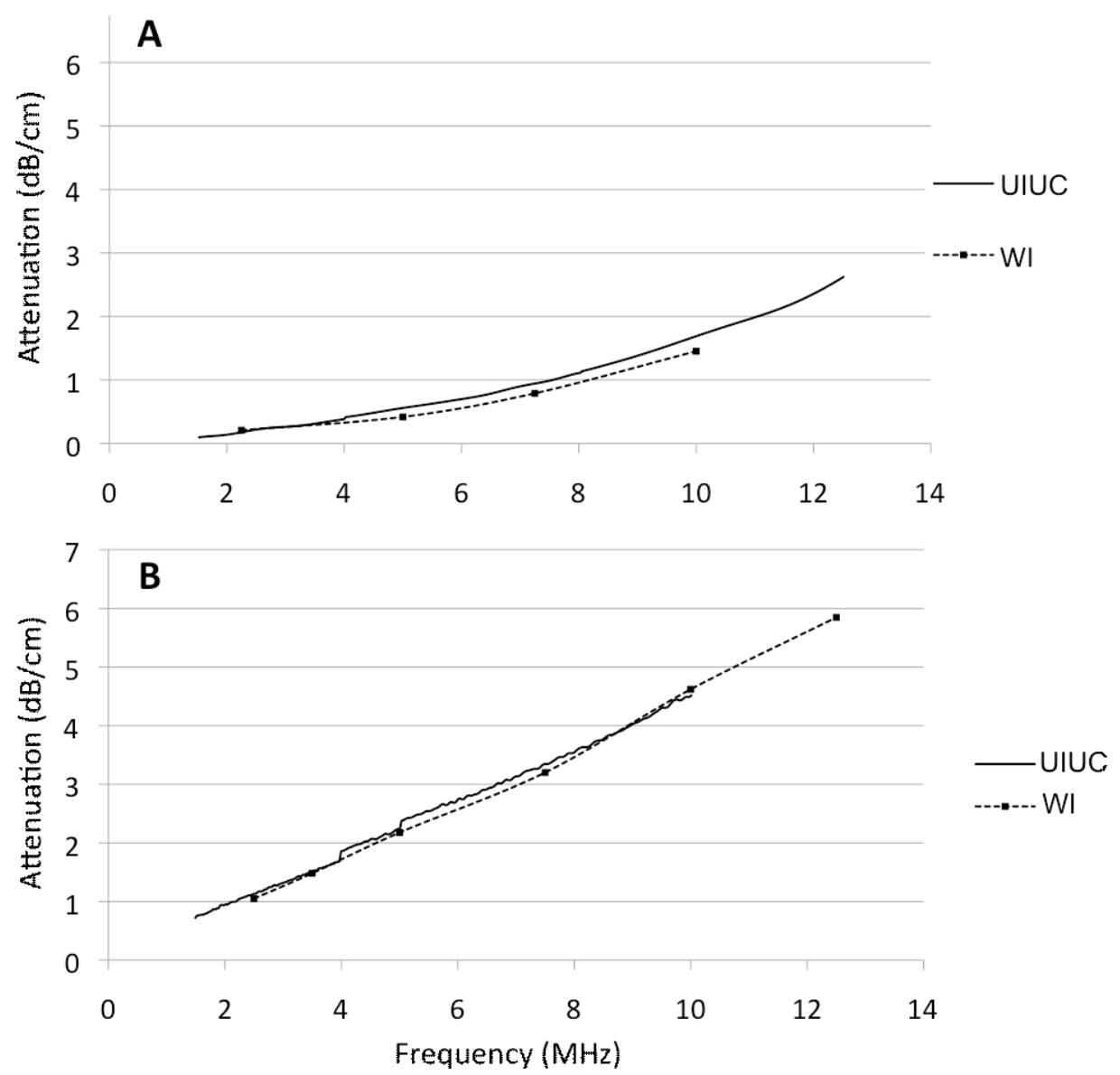

FIG. 6 Attenuation measurement comparison for the LA $41 \mu \mathrm{m}$ glass spheres in agar (A) and TLA M2 phantom (B). Standard deviations for the WI measurements were very small (around 0.02-0.08) and are not visible on these graphs.

Uncertainties in BSC estimation are dominated by the uncertainties in the power spectral density for the sample and for the planar reflector. The average uncertainty in the UW and UIUC BSC estimates are $30 \%{ }^{18}$

The best agreement among experimental estimates and Faran's theory was demonstrated with the $41 \mu \mathrm{m}$ glass spheres in the agar phantom (the most accurately-characterized glasssphere-diameter distribution), where the average fractional (bias) error relative to Faran values was $2.1 \%$ for UW and $2.6 \%$ for UIUC (Fig. 12). The average fractional (bias) error averaged over all phantoms was $8 \%$ for UW and 14\% for UIUC when compared with Faran's theory.

\section{TLA phantoms}

For the F1 and F2 phantoms, both groups' estimates agreed. Measured data were also in agreement with the Faran-predicted scattering (Fig. 7). Additionally, the elevated backscatter from different number densities among these phantoms was clearly distinguishable.

The M1, M2 and M3 phantoms all had the same Faran-predicted BSC (Figs. 8-10). Both groups' estimates are in good agreement with the theory over the measured frequency range.

For the A1 and A2 phantoms, both groups' measurements again agreed well with each other and with Faran predictions (Fig. 11). The difference in number densities between the 


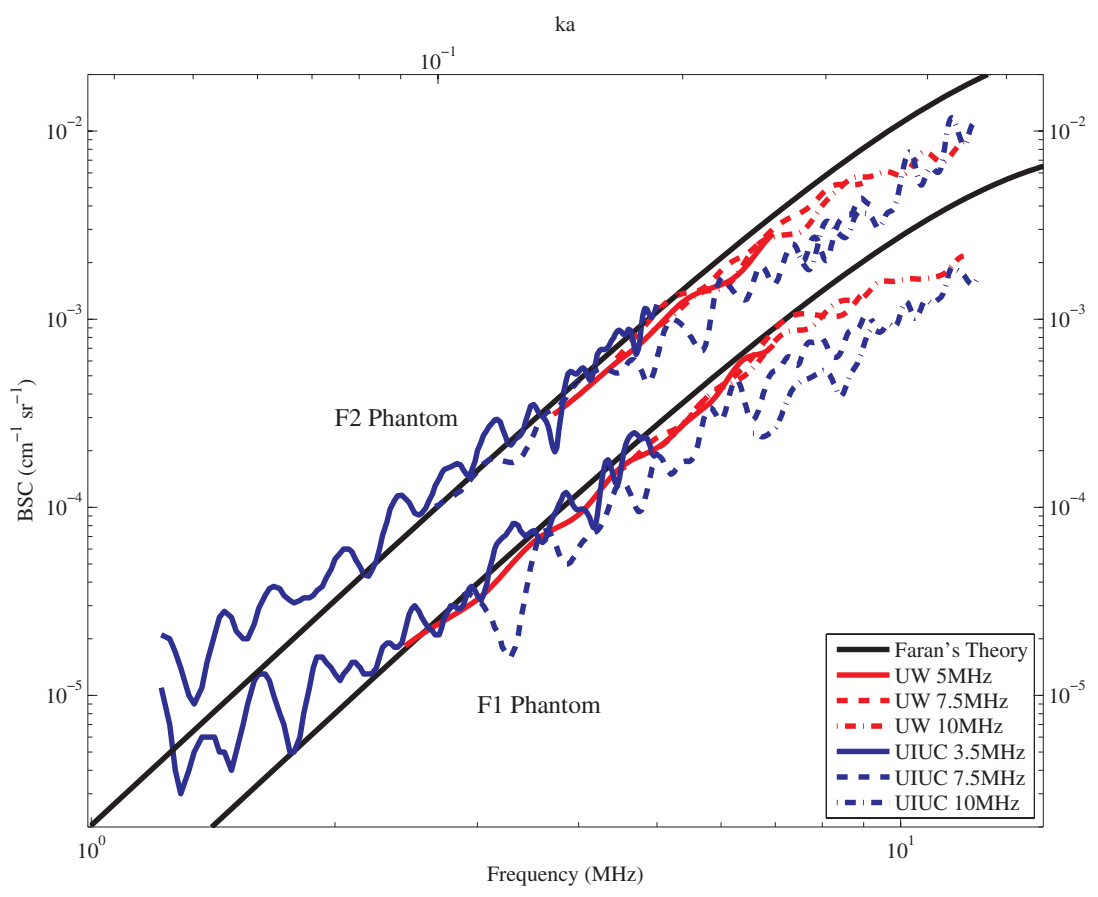

FIG. 7 BSC for the F1 and F2 TLA phantoms. The $k a$ axis was determined by using the volume-weighted mean radius of the scatterers within the phantoms.

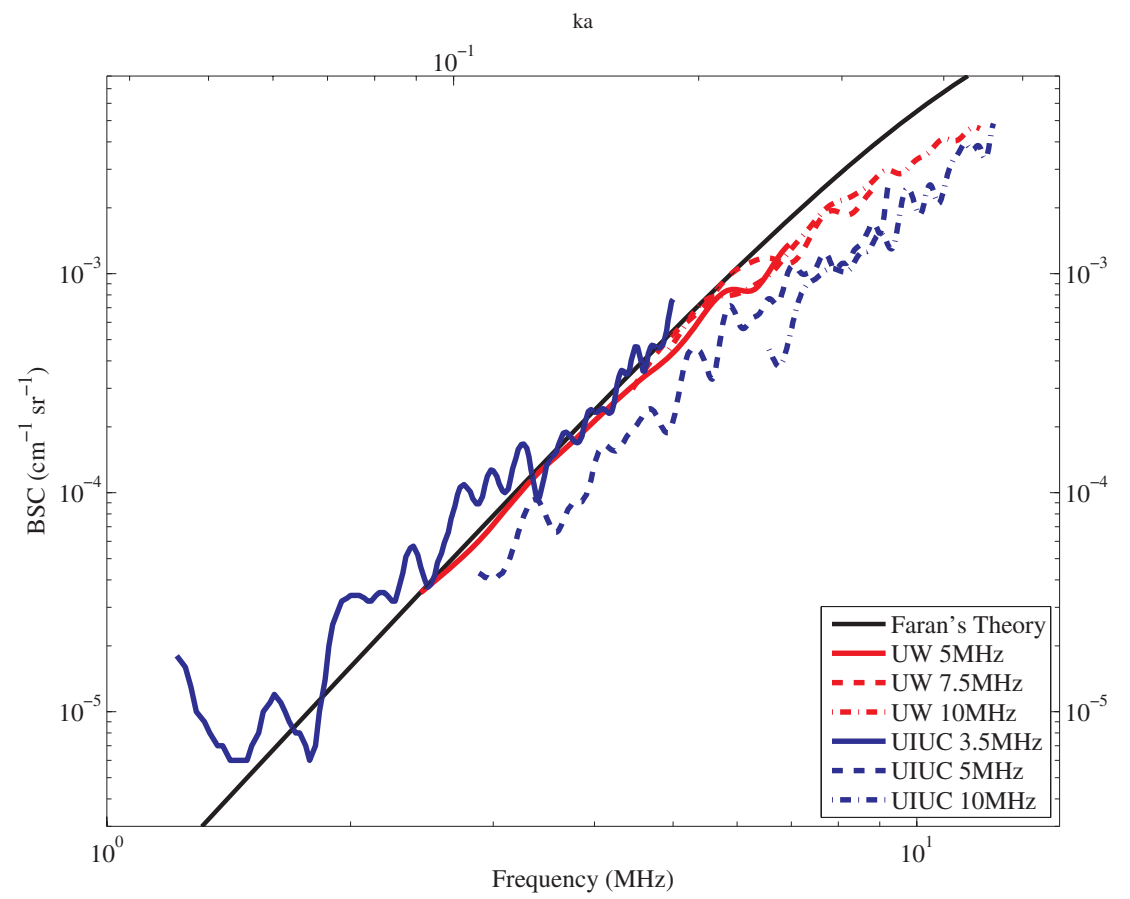

FIG. 8 BSC for the M1 TLA phantom. The $k a$ axis was determined by using the volume-weighted mean radius of the scatterers within the phantoms. 


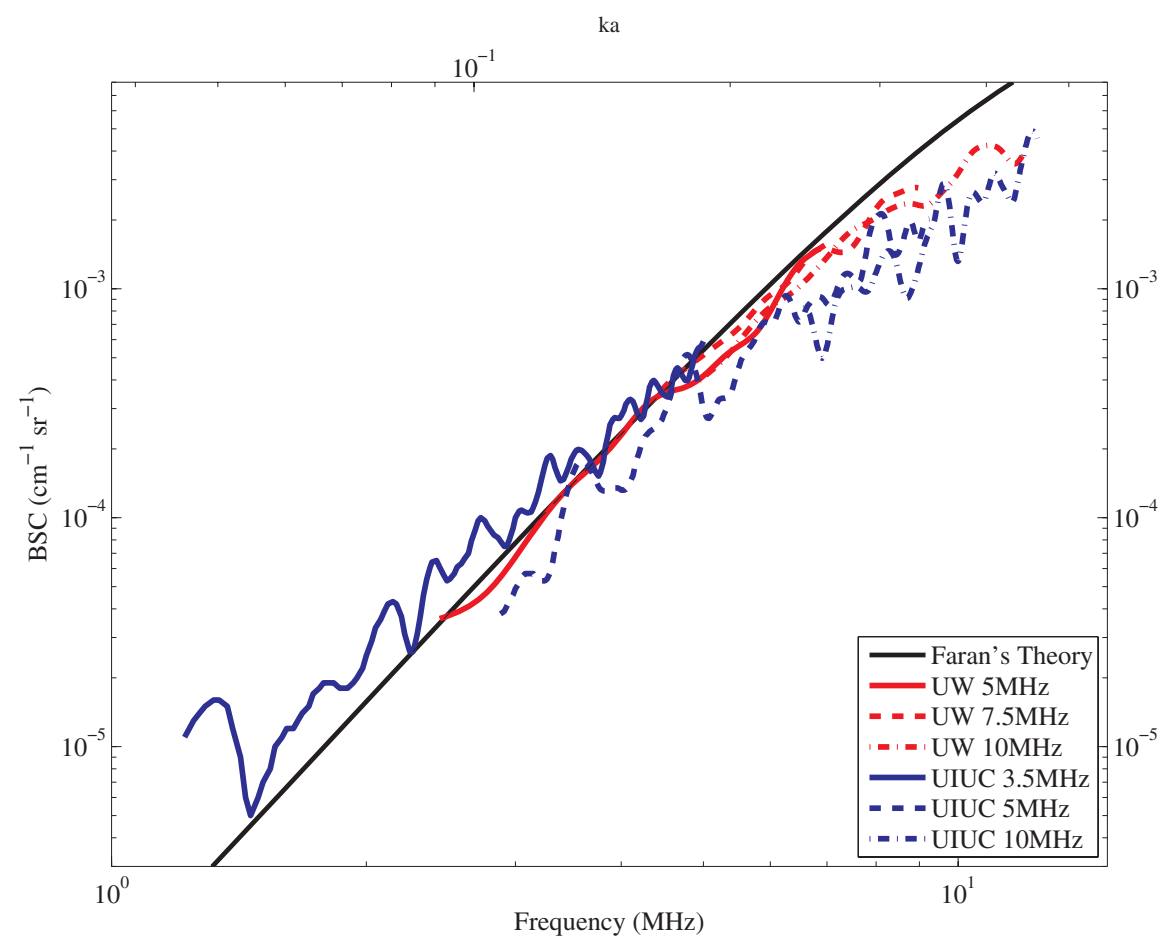

FIG.9 BSC for the M2 TLA phantom. The $k a$ axis was determined by using the volume-weighted mean radius of the scatterers within the phantoms.

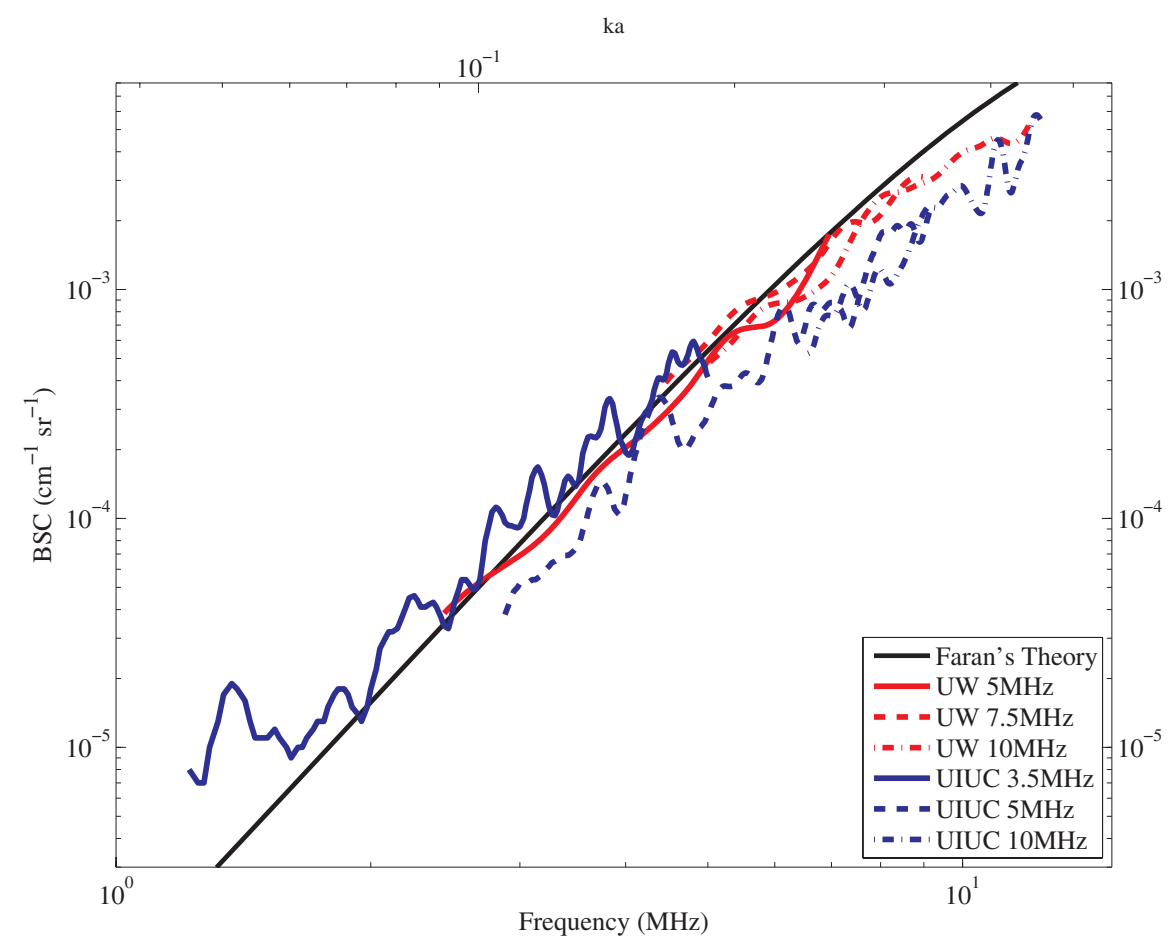

FIG. 10 BSC for the M3 TLA phantom. The $k a$ axis was determined by using the volume-weighted mean radius of the scatterers within the phantoms. 


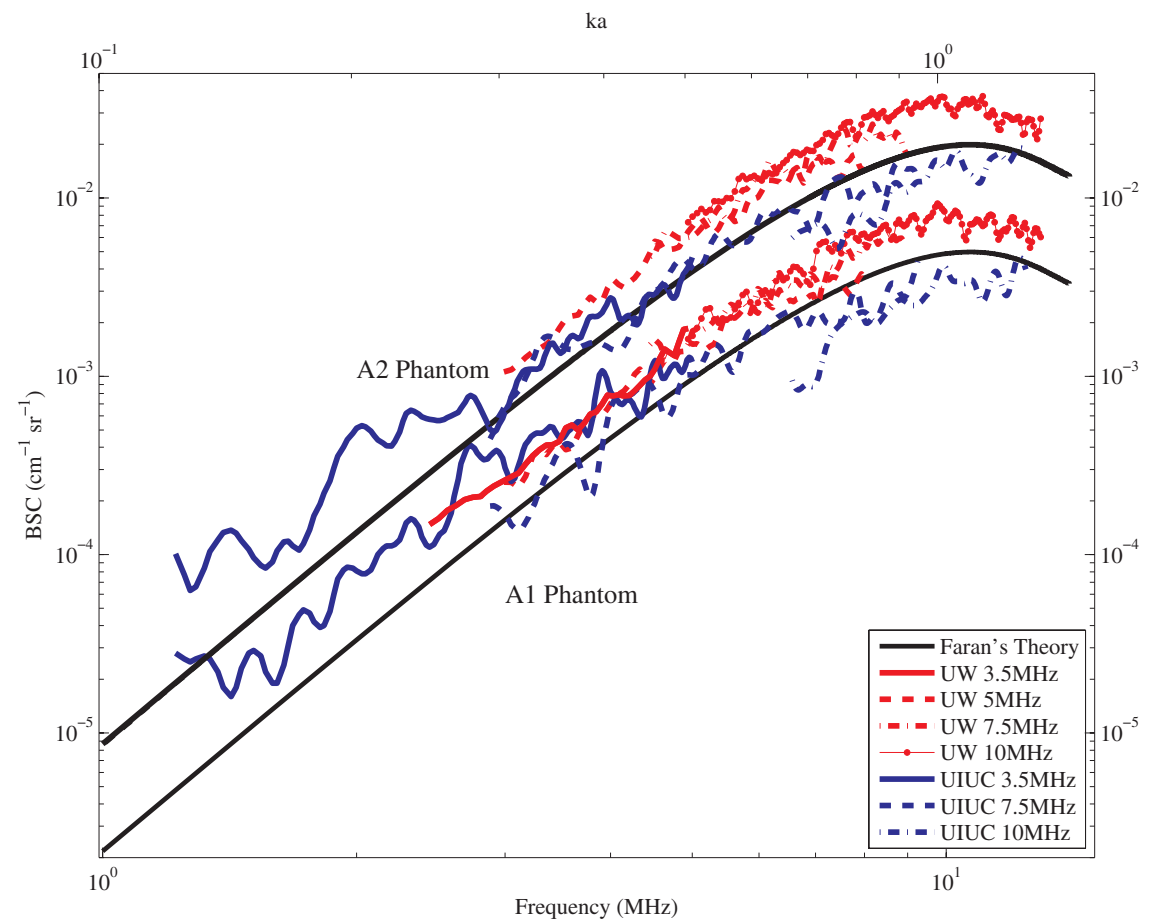

FIG. 11 BSC for the A1 and A2 TLA phantoms.

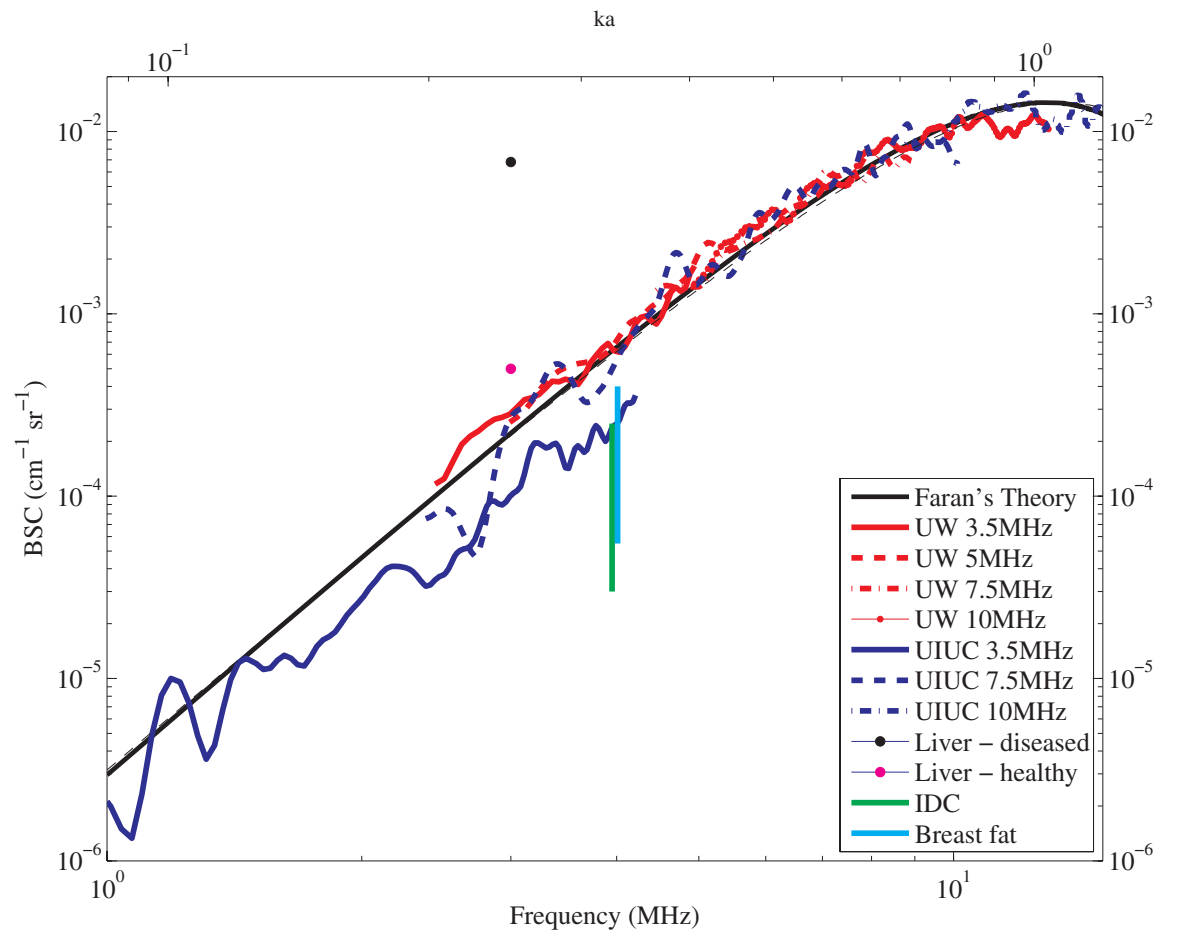

FIG. 12 BSC for the $41 \mu \mathrm{m}$ glass spheres in agar LA phantom. Single black and magenta dots represent BSC for diseased and healthy liver, respectively. ${ }^{20}$ The vertical green dotted bar represents the range of BSCs for infiltrating ductal carcinomas (IDC) and the vertical cyan dashed bar represents the range of BSCs for breast fat. ${ }^{21}$ 


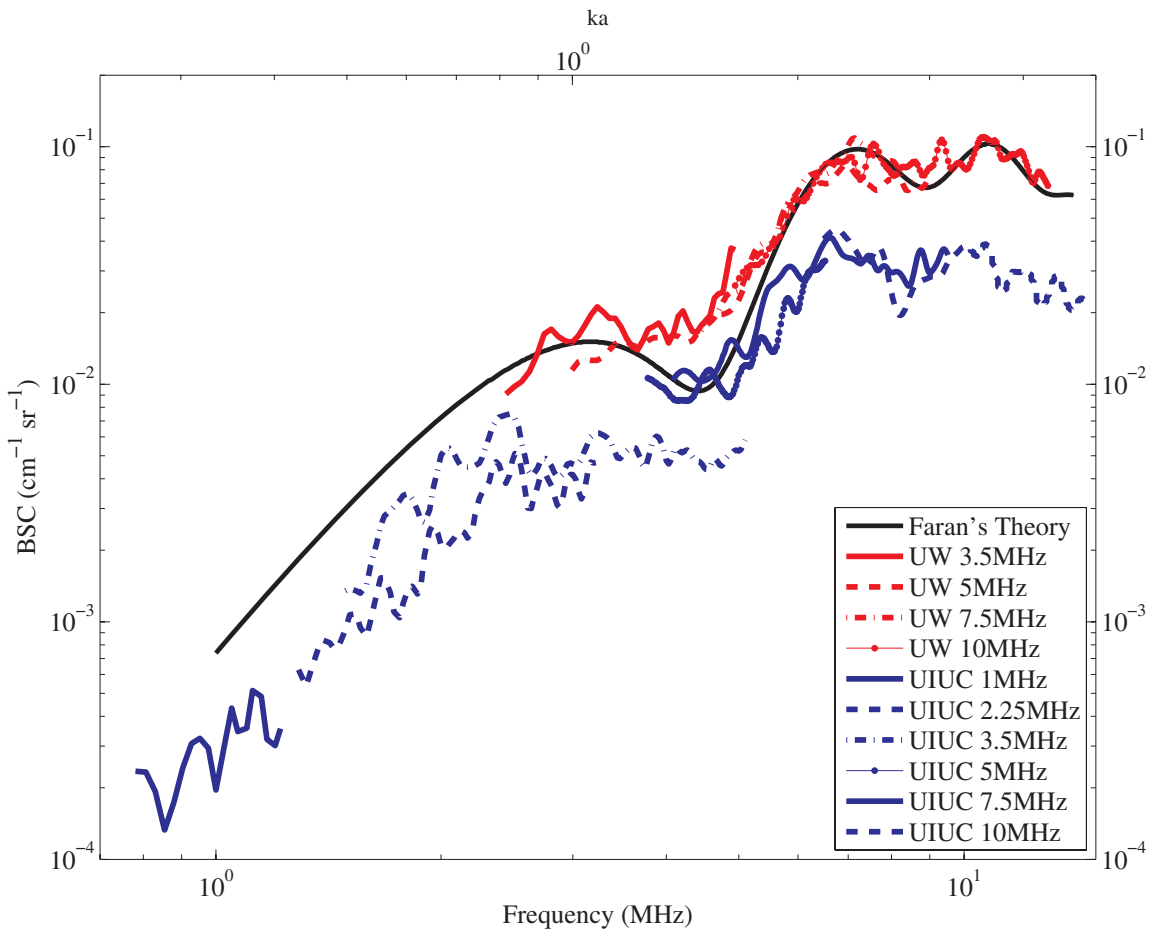

FIG. 13 BSC for the $150-180 \mu \mathrm{m}$ glass spheres in agar LA phantom.

two phantoms was visualized by the different BSC estimates and Faran predictions for each phantom.

\section{LA phantoms}

The BSC estimates for the $41 \mu \mathrm{m}$ glass spheres in agar phantom are shown in figure 12 . The best overall agreement with Faran predictions was found with this phantom where the average fractional (bias) error over the frequency range was $2.1 \%$ for UIUC and $2.6 \%$ for UW. Individual data points plotted in figure 12 compare backscatter values in this material to average BSC's determined from human tissue.

For the 150-180 $\mu$ m glass spheres in agar phantom, the UW results agreed well with Faran predictions but the UIUC results were biased low (Fig. 13). Despite this magnitude difference, both groups' frequency dependence agreed with Faran predictions.

\section{DISCUSSION}

Our results have demonstrated generally very good agreement among estimates of the important tissue characteristics of attenuation, sound speed and scattering. Some challenges remain in these measurements and more improvement is needed. The fractional uncertainty in sound speed measurements using narrowband through-transmission methods has been estimated to be less than $1 \mathrm{~m} \mathrm{~s}^{-1}$ and this is consistent with repeat measurements on the same phantom producing results within $1 \mathrm{~m} \mathrm{~s}^{-1}{ }^{19}$ The uncertainty in broadband sound speed measurements is unknown but its use may account for the discrepancies in some of the measurements in table 6 .

The reported uncertainty values in attenuation estimates were the computed standard deviations among three to five independent measurements on each sample by UW. The UIUC 
measurements generally represent single measurements for each phantom. Given the excellent agreement between attenuation estimates by these groups among these phantoms it appears that either narrowband or broadband through-transmission techniques can be used to obtain accurate results (Fig. 6).

There is generally good agreement among BSC estimates overall despite larger variations at some individual frequencies. The variance in backscatter coefficient estimates at individual frequencies is directly related to the variance in power spectral density estimates for the echo signals from the sample being investigated and that of the reference medium (e.g., planar interface or reference phantom). These (precision) errors could be reduced by increasing the number of independent lines of rf echo data used to estimate the power spectra and by repeating the measurements (instead of being measured just once by each group).

In figures 7 through 11, there is a small difference between the frequency dependence of the theoretical and measured curves. This bias error is likely due to insufficient sampling of the glass bead diameter distribution; it could be corrected by measuring the diameters of thousands (rather than the hundreds that were measured) of glass beads to more accurately capture the size distribution of such a broad distribution. Figure 12 presents the best agreement between experimental BSC estimates and Faran's theory. The glass bead scatterer size distribution in this phantom is well characterized and very narrow. There is a comparatively large bias in UIUC's 3.5 MHz transducer BSC results among these measurements but that bias error is still within the anticipated error range. In figure 13, the frequency dependence between measured results and the theory are similar, but UIUC's results are (nearly uniformly) biased low. However, these results are still within the extent of the predicted error, and additional measurements of this phantom may generate an average BSC curve closer to theory.

The liver BSC values shown in figure 12 were obtained using a reference phantom technique with a clinical ultrasound system. ${ }^{20}$ The 'diseased liver' value represents BSC measurements in patients with diffuse liver disease. That value is considerably higher magnitude than that of the healthy livers. The BSC estimate for healthy liver tissue is of comparable magnitude to the $\mathrm{BSC}$ estimates for the phantom results.

The breast tumor (infiltrating ductal carcinoma, or IDC) and the breast fat BSC values shown in figure 12 were obtained using a spherically-focused piezoelectric transmitter/receiver. ${ }^{21}$ The estimated BSC values for breast fat are slightly higher than the values for IDC but have large regions of overlap. Like the healthy liver tissue, the BSC estimates for breast fat and IDC are of comparable magnitude to the BSC estimates for the phantom results.

To extend this work, measurement techniques should be restricted to those that provide accurate sound speed and attenuation since errors in these measurements will propagate to errors in BSC estimates. Enhancing the efficiency of BSC measurements would also be a great improvement as the current techniques require many hours of measurement and data analysis to yield the presented results. Methods for sound speed and attenuation estimation with backscattered echo signals need to be compared in similar tests to those reported here. Methods, such as spatial compounding, for reducing the spatial extent of the region of interest required for estimating power spectra, need to be tested in similar interlaboratory comparisons. Furthermore, these measurements should be extended to higher frequencies to determine whether measured BSC estimates are still similar to Faran's predicted scattering (as expected).

\section{CONCLUSIONS}

With the exception of the 150-180 $\mu \mathrm{m}$ glass spheres in agar phantom, the BSC estimation methods used at UIUC and UW produced estimates over the range of 1 to $12 \mathrm{MHz}$ that 
agreed with predictions using Faran's scattering theory in frequency dependence and scattering magnitude. Also, the magnitude of the BSC estimates of these phantoms is comparable with BSC-estimates of clinically-important tissues such as the liver and breast carcinomas, as shown in figure $12.2^{20,21}$ The phantoms used in this study were constructed so their Faran predicted scattering could be easily determined. Additionally, broader distributions of scatterer diameters and higher $\mathrm{ka}$ values were used in these phantoms than were used in previous inter-laboratory comparisons.

The source of the magnitude difference between the UIUC and UW results for the $150-180 \mu \mathrm{m}$ glass spheres in agar phantom is yet to be resolved.

The accuracy of these BSC estimates will allow us to obtain more accurate BSC-derived parameters, such as effective scatterer diameter and acoustic concentration, and to use these parameters to improve characterization of tissues. Furthermore, accurate BSC estimates for various tissues will help establish a means to quantify echogenicity on an absolute scale rather than relative scales. The next stage of this work is to extend it into higher frequencies, continue validating the BSC estimation accuracy using different methods on well-characterized phantoms and continue comparisons with scattering theory.

\section{ACKNOWLEDGEMENTS}

This work supported by NIH/NCI R01CA111289.

\section{REFERENCES}

1. Madsen EL, Insana MF, Zagzebski JA. Method of data reduction for accurate determination of acoustic backscatter coefficients, J Acoust Soc Am 76, 913-923 (1984).

2. Faran JJ, Jr. Sound scattering by solid cylinders and spheres, J Acoust Soc Am 23, 405-418 (1951).

3. Burke TM, Goodsitt MM, Madsen EL, et al. Angular distribution of scattered ultrasound from a single steel sphere in agar gelatin: a comparison between theory and experiment, Ultrasonic Imaging 6, 342-347 (1984).

4. Davros WJ, Zagzebski JA, Madsen EL. Frequency-dependent angular scattering of ultrasound by tissue-mimicking materials and excised tissue, J Acoust Soc Am 80, 229-237 (1986).

5. Insana MF, Madsen EL, Hall TJ, Zagzebski JA. Tests of the accuracy of a data reduction method for determination of acoustic backscatter coefficients, J Acoust Soc Am 79, 1230-1236 (1986).

6. Hall TJ, Madsen EL, Zagzebski JA, Boote EJ. Accurate depth-independent determination of acoustic backscatter coefficients with focused transducers, J Acoust Soc Am 85, 2410-2416 (1989).

7. Insana MF, Hall TJ, Cook, LT. Backscatter coefficient estimation using array transducers, IEEE Trans Ultrason Ferroelec Freq Contr 41, 714-723 (1994).

8. Hall TJ, Insana MF, Harrison LA, Cox, GG. Ultrasonic measurement of glomerular diameters in normal adult humans, Ultrasound Med Biol 22, 987-997 (1996).

9. Boote EJ, Zagzebski JA, Madsen EL, Hall, TJ. Instrument-independent acoustic backscatter coefficient imaging, Ultrasonic Imaging 10, 121-138 (1988).

10. Boote EJ, Zagzebski JA, Madsen EL. Backscatter coefficient imaging using a clinical scanner, Med Phys 19, 1145-1152 (1992)

11. Insana MF, Hall TJ, Cook, LT. Backscatter coefficient estimation using array transducers, IEEE Trans Ultrason Ferroelec Freq Contr 41, 714-723 (1994).

12. Madsen EL, Dong F, Frank, GR, et al. Interlaboratory comparison of ultrasonic backscatter, attenuation, and speed measurements, J Ultrasound Med 18, 615-631 (1999).

13. Wear KA, Stiles TA, Frank GR, et al. Interlaboratory comparison of ultrasonic backscatter coefficient measurements from 2 to $9 \mathrm{MHz}$, J Ultrasound Med 24, 1235-1250 (2005). 
14. Madsen EL, Zagzebski JA, Banjavie RA, Jutila RE. Tissue mimicking materials for ultrasound phantoms, Med Phys 5, 391-394 (1978).

15. Hein IA, O'Brien WD, Jr. Current time-domain methods for assessing tissue motion by analysis from reflected ultrasound echoes - a review, IEEE Trans Ultrason Ferroelec Freq Contr 40, 84-102 (1993).

16. Insana MF, Hall TJ. Parametric ultrasound imaging from backscatter coefficient measurements: image formation and interpretation, Ultrason Imaging 12, 245-267 (1990).

17. Raum K, O'Brien WD, Jr. Pulse-echo field distribution measurement technique of high-frequency ultrasound sources, IEEE Trans Ultrason Ferroelec Freq Contr 44, 810-815 (1997).

18. Hall, TJ. Experimental Methods for Accurate Determination of Acoustic Backscatter Coefficients, PhD Thesis (University of Wisconsin, Madison, 1988).

19. Stiles TA, Madsen EL, Frank GR, Diehl T, Lucey JA. Tissue-mimicking liquid for use in exposimetry, JUltrasound Med 24, 501-516 (2005).

20. Lu ZF, Zagzebski JA, Lee FT. Ultrasound backscatter in human liver with diffuse disease, Ultrasound Med Biol 25, 1047-1054 (1999).

21. D'Astous FT, Foster FS. Frequency dependence of ultrasound attenuation and backscatter in breast tissue, Ultrasound Med Biol 12, 795-808 (1986). 